\title{
Generalised Absolute Stability using Lyapunov Functions with Relaxed Positivity Conditions
}

\author{
R. Drummond , G. Valmorbida and S. R. Duncan
}

\begin{abstract}
Conditions are given for verifying stability and computing upper bounds on the induced (regional) $\mathcal{L}_{2}$ gain for systems defined by vector fields which are, along with their Jacobian, rational in the states and sector bounded nonlinearities. A class of candidate Lyapunov functions is considered that are polynomial on the states and the nonlinearities and have a polynomial scaled Lurie-Postnikov term. The main result of the paper is a set of conditions that relax the requirement on the candidate Lyapunov function from being sumof-squares with respect to the nonlinearities and the Lurie-Postnikov terms from being non-negative.
\end{abstract}

\section{INTRODUCTION}

This paper studies stability and induced $\mathcal{L}_{2}$ gain for the class of systems described by the feedback structure of Figure 1, i.e. those with vector fields, which are, along with their Jacobian, rational on the state variables and sectorbounded nonlinearities. We refer to the stability analysis of this class of systems as generalised absolute stability. The original problem of absolute stability, formulated by Lurie and Postnikov [1] and summarised in [2], considered the feedback interconnection of a linear system with a generic passive nonlinear operator characterised by sector bounds. The main difference between this classical problem and the one considered here is that the nonlinear system with a rational vector field replaces the linear system in the feedback block.

To analyse such systems requires techniques from both absolute stability theory and polynomial systems. A standard technique for verifying absolute stability is the search for suitable Lyapunov functions, with classical functions being the quadratic function of the circle criterion [3] and the Lurie-Postnikov function of the Popov criterion [3]. When the nonlinearity is also slope restricted, more advanced candidate Lyapunov functions, which are quadratic on both the states and the nonlinearity and include an integral term, can also be considered $[4,5,6]$. This is the class of Lyapunov function that is generalised in this paper for the case of rational vector fields.

A generalisation of absolute stability was proposed in [7] to extend the class of systems that could be analysed

R. Drummond and S. R. Duncan are with the Department of Engineering Science, University of Oxford, 17 Parks Road, OX1 3PJ Oxford, United Kingdom, Email: $\quad$ ross.drummond,stephen.duncan\}@eng.ox.ac.uk. G. Valmorbida is with the Laboratoire des Signaux et Systèmes, CentraleSupélec, CNRS, Univ. Paris-Sud, Université ParisSaclay, 3 Rue Joliot-Curie, Gif-sur-Yvette 91192, France, Email: giorgio.valmorbida@12s.centralesupelec.fr.

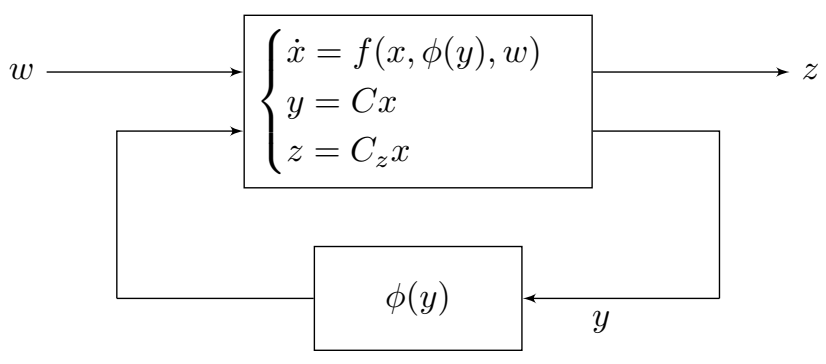

Figure 1. The feedback interconnection of the considered class of system. The vector field $f(x, \phi(y), w)$ and its Jacobian are assumed to be rational on $x$ and $\phi(y)$ and affine in $w$.

beyond those with the rather restrictive linear feedback structure. A main feature of [7] was the use of polynomials to bound the nonlinearities, with the results taking advantage of recent developments in polynomial optimization, namely the sum-of-squares (SOS) relaxation for positive polynomials. The generalisation presented in this paper proposes a refinement based on the introduction of Lyapunov/storage function candidates that contain polynomial terms and scaled integral Lurie-Postnikov terms that are not sum-of-squares. The advances in polynomial optimization also motivated the approximation of vector fields defined by (locally) analytic nonlinear functions by polynomials systems $[8,9]$. The analysis of non-polynomial systems via polynomial optimisation methods was considered in [10], which used a recasting transformation on the system.

\section{Contribution}

We propose a method to study systems defined by vector fields that are, along with their Jacobian, rational functions on both the states and sector bounded nonlinearities. The restriction on the Jacobian to be rational leads to tractable numerical solutions of polynomial Lyapunov inequalities. Moreover, we provide examples of non-linearities that are not rational, but which have a rational Jacobian. The results are based on the use of Lyapunov/storage functions that are polynomial on the states and the sector bounded nonlinearities and contain a scaled Lurie-Postnikov term. The conditions for the positivity of the Lyapunov functions extends the results for quadratic functions of [4], namely, we do not impose the Lurie-Postnikov term nor the polynomial terms on the sector nonlinearities to be strictly positive. The prescribed analysis also encompasses regional stability and 
performance, with inclusion conditions on the level sets of the Lyapunov function being used to obtain local properties. More importantly, the conditions presented are expressed in terms of the verification of polynomial inequalities, which are cast as semi-definite programmes, convex optimisation problems that can be efficiently solved for low dimensional systems using software packages such as YALMIP or SOSTOOLS.

A preliminary version of the material in this paper can be found in [11], where only a global analysis was considered and a simpler version of the Lyapunov function than the one studied here was proposed. Note that since the vector field is defined by rational expressions and not by a linear system, it is not possible to formulate the frequency dependent interpretation of the stability conditions, such as phase equivalent Zames-Falb multipliers [12], which are generally considered to be the least conservative method for the global analysis of absolute stability [12].

The paper is structured as follows. Section I sets up the system to be studied. Section II introduces a set of inequalities for verifying stability and computing upper bounds on the induced $\mathcal{L}_{2}$ gain from input to output of the system in some local/global domain. Section III details a numerical formulation for the inequalities such that they can be verified using convex programming. Numerical examples illustrate the proposed method in Section IV. The first numerical example shows the existence of a common Lyapunov function for a benchmark saturated system while the second example considers the stability of a nonlinear vehicle model.

Notation: The $i^{\text {th }}$ row of matrix $C \in \mathbb{R}^{p \times n}$ is denoted $C_{i}$ and the $i^{\text {th }}$ element of vector $y$ is denoted $y_{i}$. A polynomial $\mathcal{R}[x]$ is a function that can be expressed as $p(x)=p_{1} x+\cdots+p_{m} x^{m}$. Polynomials of real coefficients of dimension $n$ in variable $\theta$ are denoted $\mathcal{R}^{n}[\theta]$. We use $\mathcal{R}[\theta]$ for $n=1$. Polynomials on two variables $\theta_{1}$ and $\theta_{2}$ are denoted $\mathcal{R}^{n}\left[\left(\theta_{1}, \theta_{2}\right)\right]$. The positive polynomials of dimension $n$ are denoted $\mathcal{R}_{>0}^{n}\left[\left(\theta_{1}, \theta_{2}\right)\right]$ and the non-negative polynomials are $\mathcal{R}_{\geq 0}^{n}\left[\left(\theta_{1}, \theta_{2}\right)\right]$. The positive sum-of-squares polynomials of dimension $n$ are denoted $\Sigma^{n}\left[\left(\theta_{1}, \theta_{2}\right)\right]$. The diagonal sum-of-squares polynomials are $\Sigma_{n}^{\text {diag }}\left[\left(\theta_{1}, \theta_{2}\right)\right]$. For $p \in \mathcal{R}[\theta], \operatorname{deg}(p, \theta)$ stands for the degree of $p$ on variable $\theta$. The set of non-negative reals is denoted $\mathbb{R}_{\geq 0}$. The set of real symmetric matrices of dimension $n$ is denoted $\mathbb{S}^{n}$ and the subset of diagonal matrices is denoted $\mathbb{D}^{n}$. The sets of positive semidefinite symmetric and diagonal matrices are respectively $\mathbb{S}_{>0}^{n}$ and $\mathbb{D}_{>0}^{n}$. The set of real positive definite matrices are $\mathbb{S}_{>0}^{n}$ and $\mathbb{D}_{>0}^{n}$. The vector of dimension $n$ containing zeros is denoted $\mathbf{0}^{n}$. The $\mathcal{L}_{2}$ norm of a signal is denoted $\|z\|_{2}=\sqrt{\int_{0}^{\infty} z^{T}(\theta) z(\theta) \mathrm{d} \theta}$. We define the $\rho$ sublevel set of $V$ by $\mathcal{E}(V, \rho)=\left\{x \in \mathbb{R}^{n} \mid V(x, \phi) \leq \rho\right\}$ for $\rho \geq 0$. The time derivative of a function $V(x, \phi): \mathcal{X}_{0} \rightarrow$ $\mathbb{R}_{\geq 0}$ is denoted by the inner product $\dot{V}=\frac{d V(x, \phi)}{d x} \dot{x}+\frac{d V}{d \phi} \dot{\phi}=$ $\dot{V}(x, \phi)=\left\langle\left[\begin{array}{c}\nabla_{x} V \\ \nabla_{\phi} V\end{array}\right],\left[\begin{array}{l}\dot{x} \\ \dot{\phi}\end{array}\right]\right\rangle$. In a slight abuse of notation, we may drop the argument of functions, most notably with $\phi(y(x))=\phi$ and $\phi_{i}\left(y_{i}(x)\right)=\phi_{i}$.

\section{Problem Statement}

Consider the dynamical system

$$
\begin{aligned}
\dot{x} & =D_{1}(x, \phi)^{-1} N_{1}(x, \phi)+B_{w} w \\
y & =C x \\
z & =C_{z} x
\end{aligned}
$$

with $x \in \mathbb{R}^{n}, y \in \mathcal{Y} \subseteq \mathbb{R}^{m}, \phi(y): \mathcal{Y} \rightarrow \mathbb{R}^{m}, \phi \in \mathcal{C}^{1}$, output $z \in \mathbb{R}^{n_{z}}$, disturbance $w \in \mathbb{R}^{n_{w}}, N_{1} \in \mathcal{R}^{n}[(x, \phi)]$, $D_{1} \in \mathcal{R}_{>0}[(x, \phi)]$. The nonlinearity $\phi(y(x))$ is defined on $\mathcal{Y}$, which contains the origin and a domain $\mathcal{Y}_{0}$ satisfying $\mathcal{Y}_{0} \subseteq \mathcal{Y} \subseteq \mathbb{R}^{m}$. Define the set $\mathcal{X}_{0}$ as $\mathcal{X}_{0}=\left\{x \mid y(x) \in \mathcal{Y}_{0}\right\}$. This system can be interpreted by the feedback form of Figure 1 with $f(x, \phi(y), w)$ defined by the RHS of (1a).

We will restrict the considered class of nonlinearities to those which are memoryless, static, decentralised

$$
\phi(y)=\left[\begin{array}{llll}
\phi_{1}\left(y_{1}\right), & \phi_{2}\left(y_{2}\right), & \ldots, & \phi_{m}\left(y_{m}\right)
\end{array}\right]^{T},
$$

sector bounded with the nonlinearities $\phi_{i}$ being signpreserving operators on $y_{i}$ satisfying

$$
\frac{\phi_{i}\left(y_{i}\right)}{y_{i}} \in\left[0, \overline{\delta_{i}}\right] \quad \forall y \in \mathcal{Y}_{0} \subseteq \mathcal{Y}
$$

and

$$
\frac{\mathrm{d} \phi_{i}\left(y_{i}\right)}{\mathrm{d} y_{i}}=D_{2 i}\left(y_{i}, \phi_{i}\right)^{-1} N_{2 i}\left(y_{i}, \phi_{i}\right),
$$

where $N_{2 i} \in \mathcal{R}\left[\left(y_{i}, \phi_{i}\right)\right]$ and $D_{2 i} \in \mathcal{R}_{>0}\left[\left(y_{i}, \phi_{i}\right)\right]$. Defining $\bar{D}_{2}(y, \phi) \in \mathcal{R}_{>0}[(y, \phi)]$ and $\bar{N}_{2}(y, \phi)=\operatorname{diag}\left(\bar{N}_{21}, \ldots, \bar{N}_{2 m}\right)$ as

$$
\begin{aligned}
\bar{D}_{2}(y, \phi) & =\prod_{i=1, \ldots, m} D_{2 i}\left(y_{i}, \phi_{i}\right) \\
\bar{N}_{2 i}(y, \phi) & =N_{2 i}\left(y_{i}, \phi_{i}\right) \prod_{\substack{j=1, \ldots, m \\
i \neq j}} D_{2 j}\left(y_{j}, \phi_{j}\right)
\end{aligned}
$$

such that

$$
D_{2 i}^{-1}\left(y_{i}, \phi_{i}\right) N_{2 i}\left(y_{i}, \phi_{i}\right)=\bar{D}_{2}^{-1}(y, \phi) \bar{N}_{2 i}(x, \phi)
$$

gives

$$
\frac{d \phi(y)}{d y}=\bar{D}_{2}(y, \phi)^{-1} \bar{N}_{2}(y, \phi) .
$$

The reason for introducing $\bar{D}_{2}(y, \phi)$ and $\bar{N}_{2}(y, \phi)$ is to allow the denominator terms to be cancelled out in the stability conditions.

The sector boundedness of the nonlinearity can be used to obtain a polynomial inequality, known as the sector inequality, that relates $y$ and $\phi(y)$.

Fact 1: If the sector condition of (2b) holds, then

$$
s_{1}(y, \phi, T(x, \phi)):=\phi^{T} T(x, \phi)(\bar{\Delta} y-\phi) \geq 0 \forall y \in \mathcal{Y}_{0}
$$

where $T=\operatorname{diag}\left(\tau_{1}, \tau_{2}, \ldots, \tau_{m}\right)$ with $\tau_{i} \in \mathcal{R}_{\geq 0}[(x, \phi)]$ for $i=1,2, \ldots, m$ and $\bar{\Delta}=\operatorname{diag}\left(\bar{\delta}_{1}, \ldots, \bar{\delta}_{m}\right)$.

The sector inequalities relating $\phi(y)$ and its argument are used to verify the positivity of polynomials in $y$ and $\phi(y)$ as in the fact below. 
Fact 2: Consider a polynomial $p_{1} \in \mathcal{R}[(y, \phi)]$. If the inequality

$$
p_{1}(y, \phi)-s_{1}(y, \phi, T(x, \phi)) \geq 0 \forall x \in \mathcal{X}_{0}
$$

holds then $p_{1}(y, \phi) \geq 0 \forall x \in \mathcal{X}_{0}$.

This paper proposes solutions to the following problems:

Problem 1 (Stability): Provide conditions that guarantee local stability of the origin of system (1).

Problem 2 ( $\mathcal{L}_{2}$ gain): Obtain (local) upper bounds for the induced $\mathcal{L}_{2}$ gain from $w$ to $z$ of $(1)$.

\section{Main Results}

This section gives a set of conditions in terms of inequalities to solve Problems 1 and 2. The analysis is based upon Lyapunov function candidates of the form

$$
V(x, \phi)=V_{0}(x, \phi)+\sum_{i=1}^{m} \int_{0}^{y_{i}} \lambda_{i}(\sigma) \phi_{i}(\sigma) d \sigma
$$

where $V_{0} \in \mathcal{R}[(x, \phi)], V_{0}(0,0)=0$, and $\lambda_{i} \in \mathcal{R}[\sigma], i=$ $1, \ldots, m$. The integral term in (9) is referred to as the Lurie-Postnikov term.

Using (7), a polynomial lower bound for $V(x, \phi)$ is given by the following lemma:

Lemma 1: Let $V_{0} \in \mathcal{R}[(x, \phi)]$ and $\lambda_{i} \in \mathcal{R}[\sigma]$ for $i=$ $1, \ldots, m$ defining $(9)$ be given and let $\mathcal{X}_{0} \subseteq \mathbb{R}^{n}, 0 \in \mathcal{X}_{0}$ with $\phi$ satisfying (2). If there exists $\tilde{\lambda}_{i} \in \mathcal{R}_{\geq 0}[\sigma]$ for $i=$ $1, \ldots, m$, such that

$$
\begin{aligned}
\lambda_{i}(\sigma) \geq & -\tilde{\lambda}_{i}(\sigma), i=1, \ldots, m, \quad \forall x \in \mathcal{X}_{0} \\
\underline{V}(x, \phi) & =V_{0}(x, \phi) \\
& -\sum_{i=1}^{m} \int_{0}^{y_{i}(x)} \bar{\delta}_{i} \sigma \tilde{\lambda}_{i}(\sigma) d \sigma>0 \forall x \in \mathcal{X}_{0},
\end{aligned}
$$

where $\bar{\delta}_{i}$ is the sector bound, then $V(x, \phi)>0 \forall x \in$ $\mathcal{X}_{0} \backslash\{0\}$.

Proof: We use (10) and the sector bounds (2b) to obtain that $V(x, \phi)$ is lower bounded by $\underline{V}(x)$ as follows

$$
\begin{aligned}
V(x, \phi)= & V_{0}(x, \phi)+\sum_{i=1}^{m} \int_{0}^{y_{i}(x)} \lambda_{i}(\sigma) \phi_{i}(\sigma) \mathrm{d} \sigma \\
\geq & V_{0}(x, \phi)-\sum_{i=1}^{m} \int_{0}^{y_{i}(x)} \tilde{\lambda}_{i}(\sigma) \phi_{i}(\sigma) \mathrm{d} \sigma \\
= & V_{0}(x, \phi)-\sum_{i=1}^{m} \int_{0}^{y_{i}(x)} \tilde{\lambda}_{i}(\sigma) \phi_{i}(\sigma) \mathrm{d} \sigma \\
& -\sum_{i=1}^{m} \int_{0}^{y_{i}(x)} \bar{\delta}_{i} \sigma \tilde{\lambda}_{i}(\sigma) \mathrm{d} \sigma \\
& +\sum_{i=1}^{m} \int_{0}^{y_{i}(x)} \frac{\delta_{i}}{\delta_{i}} \tilde{\lambda}_{i}(\sigma) \mathrm{d} \sigma \\
= & V_{0}(x, \phi)-\sum_{i=1}^{m} \int_{0}^{y_{i}(x)} \overline{\delta_{i}} \sigma \tilde{\lambda}_{i}(\sigma) \mathrm{d} \sigma \\
& +\sum_{i=1}^{m} \int_{0}^{y_{i}(x)} \tilde{\lambda}_{i}(\sigma)\left(\overline{\delta_{i}} \sigma-\phi_{i}(\sigma)\right) \mathrm{d} \sigma \\
\geq & \underline{V}(x, \phi) .
\end{aligned}
$$

The following gives a set of inequalities that use the lower bound of Lemma 1 to verify solutions to Problems 1 and 2. Note that the term

$$
p_{l}(x):=\sum_{i=1}^{m} \int_{0}^{y_{i}(x)} \bar{\delta}_{i} \sigma \tilde{\lambda}_{i}(\sigma) d \sigma
$$

in (10b) is a polynomial $P_{l} \in \mathcal{R}[x]$.
Theorem 1: Suppose there exists polynomials $V_{0} \in$ $\mathcal{R}[(x, \phi)], T_{j}=\operatorname{diag}\left(\tau_{j 1}, \tau_{j 2}, \ldots, \tau_{j m}\right) j=1,2$ with $\tau_{j,} \in \mathcal{R}_{\geq 0}[(x, \phi)], \tilde{\lambda}_{i} \in \mathcal{R}_{\geq 0}[\sigma], \lambda_{i} \in \mathcal{R}[\sigma], i=1,2, \ldots, m$ and a scalar $\eta \in \mathbb{R}_{>0}$ such that (10a) and

$$
\begin{aligned}
& \underline{V}(x, \phi)-s_{1}\left(x, \phi, T_{1}(x, \phi)\right) \geq 0 \forall x \in \mathcal{X}_{0}, \\
& -\left\langle\left[\begin{array}{c}
\nabla_{x} V \\
\nabla_{\phi} V
\end{array}\right],\left[\begin{array}{c}
\dot{x} \\
\dot{\phi}
\end{array}\right]\right\rangle-\Omega(z, w) \\
& \quad-s_{1}\left(x, \phi, T_{2}(x, \phi)\right)>0 \forall x \in \mathcal{X}_{0}, \\
& \mathcal{E}(V, \rho) \subseteq \mathcal{X}_{0}
\end{aligned}
$$

hold with

a) $\Omega(z, w)=0$ and $w=0$;

b) $\Omega(z, w)=-w^{T} w+\eta^{-1} z^{T} z$;

then

a) The origin of (1) is locally asymptotically stable with domain of attraction $\mathcal{E}(V, \rho)$.

b) The induced $\mathcal{L}_{2}$ gain from $w$ to $z$ is upper-bounded by $\sqrt{\eta}$, that is, $\frac{\|z\|_{2}}{\|w\|_{2}} \leq \sqrt{\eta}$ for all solutions of (1) satisfying $x(0)=0$ and $\|w\|_{2} \leq \rho^{\frac{1}{2}}$.

Proof: From Fact 2, if (13a) holds, then $\underline{V}(x)>$ $0 \forall x \in \mathcal{X}_{0}$. Positivity of $V(x, \phi)>0$ then follows from Lemma 1 . Denote by $\dot{V}(x)$ the time derivative of the Lyapunov function $V(x, \phi)$ along the trajectories of (1) such that $\dot{V}(x, \phi)=\left\langle\left[\begin{array}{l}\nabla_{x} V \\ \nabla_{\phi} V\end{array}\right],\left[\begin{array}{l}\dot{x} \\ \dot{\phi}\end{array}\right]\right\rangle$. Invoking Fact 2, if (13b) holds, then

$$
-\dot{V}(x, \phi)-\Omega(z, w)>0 \forall x \in \mathcal{X}_{0} .
$$

With

a) $\Omega(z, w)=0$, then (14) implies $\dot{V}(x, \phi)<0 \forall x \in \mathcal{X}_{0}$. The set inclusion condition (13c) enforces the containment of the sublevel sets of the Lyapunov function $\mathcal{E}(V, \rho)$ in the region where the local sector holds. The satisfaction of (13a) and (13b) implies positive invariance and contraction of the sublevel sets $\mathcal{E}(V, \rho)$ which define an inner estimate of the region of attraction of the system.

b) $\Omega(z, w)=-w^{T} w+\eta^{-1} z^{T} z$, then integrating (14) from 0 to $t^{*}$ with $x(0)=0$ gives $\int_{0}^{t^{*}} w^{T}(\tau) w(\tau) d \tau>$ $\eta^{-1} \int_{0}^{t^{*}} z^{T}(\tau) z(\tau) d \tau+V\left(x\left(t^{*}\right)\right)$. For $\|w\|_{2} \leq \rho^{\frac{1}{2}}$, we obtain $\rho>\eta^{-1} \int_{0}^{t^{*}} z^{T}(\tau) z(\tau) d \tau+V\left(x\left(t^{*}\right)\right) \geq$ $V\left(x\left(t^{*}\right)\right)$, that is $x\left(t^{*}\right) \in \mathcal{E}(V, \rho)$, thus from (13c) it follows that $x\left(t^{*}\right) \in \mathcal{X}_{0}$ for $t^{*} \in[0, \infty)$. Therefore, with $\|w\| \leq \rho^{\frac{1}{2}},(13 \mathrm{~b})$ and (13c) hold true and $\int_{0}^{t^{*}} w(\tau)^{T} w(\tau) d \tau \geq \eta^{-1} \int_{0}^{t^{*}} z(\tau)^{T} z(\tau) d \tau$ that is $\frac{\|z\|_{2}}{\|w\|_{2}} \leq \sqrt{\eta}$.

Remark 1: Note that Theorem 1 does not require strict positivity of the polynomials $\lambda_{i}$, nor of the terms of $V_{0}$ related to the variables $\phi$. Positivity of the nonlinear terms of $V_{0}$ is relaxed by including the sector information into (13a) while the positivity of $\lambda_{i}$ is relaxed by using the polynomial lower bound of the Lurie-Postnikov term given in Lemma 1. 


\section{Numerical Formulation}

In this section, we propose numerical relaxations to solve the polynomial inequality constraints of Theorem 1 . These relaxations use the SOS formulation for positive polynomials, which is briefly explained in the appendix. See [13] for a comprehensive analysis.

The set containment condition in (13c) can be cast as a polynomial inequality in the case when the LuriePostnikov term is itself a polynomial. Otherwise, we verify (13c) by relying on (10b) and use a polynomial outer approximation of $\mathcal{E}(V, \rho)$. This outer approximation is obtained by noting from (10b) that $\underline{V}(x, \phi) \leq V(x, \phi)$, thus $\mathcal{E}(V, \rho) \subseteq \mathcal{E}(\underline{V}, \rho)$.

To formulate (13) as polynomial inequalities, in this section we assume $\mathcal{X}_{0}$ to be a semi-algebraic set, that is $\exists g_{i}(x) \geq 0, i=1, \ldots, n_{p}$ such that $\mathcal{X}_{0}=\left\{x \in \mathbb{R}^{n} \mid g_{i}(x) \geq\right.$ $\left.0, i=1, \ldots, n_{p}\right\}$. In the proposition below, we denote $g(x)=\left[g_{1}(x), \ldots, g_{n_{p}}(x)\right]$.

The following proposition gives a convex formulation of Theorem 1, expressed as an SDP.

Proposition 1: If there exists $V_{0} \in \mathcal{R}[(x, \phi)]$, $V_{0}(0,0)=0, \quad T_{1}, \quad \bar{T}_{2} \in \Sigma_{m}^{\text {diag }}[(x, \phi)], \quad \lambda_{i}(\sigma) \in \mathcal{R}[\sigma]$, $\tilde{\lambda}_{i}(\sigma) \in \Sigma[\sigma]$, satisfying $\lambda_{i}(\sigma)+\tilde{\lambda}_{i}(\sigma) \in \Sigma[\sigma]$ for $i=1, \ldots, m, q_{j} \in \Sigma^{n_{p}}[(x, \phi)]$ for $j=1, \ldots, 3, \eta \in \mathbb{R}_{>0}$, $\epsilon>0$,

$\underline{V}(x, \phi)-s_{1}\left(y(x), \phi, T_{1}\right)-\varepsilon x^{T} x-q_{1}^{T}(x, \phi) g(x, \phi) \in \Sigma[(x, \phi)]$,

$-\left(\left\langle\left[\begin{array}{c}\nabla_{x} V \\ \nabla_{\phi} V\end{array}\right],\left[\begin{array}{c}\left.D_{1}(x, \phi)\right)^{-1} N_{1}(x, \phi)+B_{w} w \\ \bar{D}_{2}(\phi, y)^{-1} \bar{N}_{2}(\phi, y) \dot{y}(x)\end{array}\right]\right\rangle\right.$

$-\Omega(w, z)) \cdot\left(D_{1}(x, \phi) \bar{D}_{2}(x, \phi)\right)-s_{1}\left(y, \phi, T_{2}\right)$

$-q_{2}^{T}(x, \phi) g(x, \phi)-\varepsilon x^{T} x \in \Sigma[(x, \phi, w)]$,

$\rho-\underline{V}(x, \phi)-q_{3}^{T}(x, \phi) g(x, \phi) \in \Sigma[x]$,

then

a) $\Omega(w, z)=0$ and $w=0$, the origin of (1) is locally asymptotically stable with domain of attraction $\mathcal{E}(V, \rho)$.

b) $\Omega(z, w)=w^{T} w-\eta^{-1} z^{T}(x) z(x)$ an upper-bound for the induced $\mathcal{L}_{2}$ gain of (1) is given by $\sqrt{\eta}$ for all solutions of (1) satisfying $x(0)=0, x(t) \in \mathcal{X}_{0}$ and $\|w\| \leq \rho^{\frac{1}{2}}$.

Proof:

We show that (15) implies (13). From (15a), it follows that $\underline{V}(x)-s_{1}\left(y(x), \phi, T_{1}\right) \geq \varepsilon x^{T} x+q_{1}^{T}(x, \phi) g(x, \phi)$. Since $g_{j}(x, \phi) \geq 0 \forall x \in \mathcal{X}_{0}$, we obtain (13a).

Similarly, (15b) implies

$$
\begin{aligned}
& \left(\left\langle\left[\begin{array}{c}
\nabla_{x} V \\
\nabla_{\phi} V
\end{array}\right],\left[\begin{array}{c}
D_{1}(x, \phi)^{-1} N_{1}(x, \phi)+B_{w} w \\
\bar{D}_{2}(\phi, y)^{-1} \bar{N}_{2}(\phi, y) \dot{y}
\end{array}\right]\right\rangle\right. \\
& -\Omega(w, z)) \cdot\left(D_{1}(\phi, y) \bar{D}_{2}(\phi, y)\right) \geq 0 \forall x \in \mathcal{X}_{0} .
\end{aligned}
$$

Dividing the above by the positive polynomial $\left(D_{1}(x, \phi) \bar{D}_{2}(x, \phi)\right)$ we obtain (14), which is Fact 2 applied to (13b).

Finally, (15c) implies $\rho-\underline{V}(x, \phi) \geq 0 \forall x \in \mathcal{X}_{0}$, thus $\mathcal{E}(\underline{V}, \rho) \subseteq \mathcal{X}_{0}$ and since $\mathcal{E}(V, \rho) \subseteq \mathcal{E}(\underline{V}, \rho),(13 \mathrm{c})$ holds.

Remark 2: For quadratic formulations $V(x, \phi)=x^{T} P x$, a convex optimization problem that enlarges the estimate of the region of attraction is obtained using the function $\log (\operatorname{det}(P))$. For the enlargement of the estimate of $\mathcal{E}(V, \rho)$ used here, one can rely on a sequence of solutions to SDPs optimising the ERA as proposed in [14].

\section{EXAMPLES}

A function satisfying (2b) and (2c) globally (with $\mathcal{Y}=\mathbb{R})$ is the $\tan ^{-1}(y)$ function. Indeed, in this case, $(2 \mathrm{~b})$ holds with $\bar{\delta}=1$ and the slope constraint (2c) holds since

$$
\frac{d \tan ^{-1}(y)}{d y}=\frac{1}{1+y^{2}} \in[0,1) .
$$

The function $\tan ^{-1}(y)$ can be regarded as a smooth approximation of the saturation function and also appears in applications, as detailed in the example below.

\section{A. Numerical Example 1: Smooth Saturation}

Consider the Lurie-Postnikov system

$$
\begin{aligned}
{\left[\begin{array}{l}
\dot{x}_{1} \\
\dot{x}_{2}
\end{array}\right] } & =\left[\begin{array}{cc}
-0.05 & 1 \\
-10 & -0.5
\end{array}\right]\left[\begin{array}{l}
x_{1} \\
x_{2}
\end{array}\right]+\left[\begin{array}{l}
0 \\
1
\end{array}\right] \tan ^{-1}(y), \\
y & =\left[\begin{array}{ll}
9.9 & 0.495
\end{array}\right]\left[\begin{array}{l}
x_{1} \\
x_{2}
\end{array}\right] .
\end{aligned}
$$

The above system is adapted from [15, Example 4.3.1] with the difference being that smooth saturation is considered here, with the non-differentiable saturation function approximated by $\tan ^{-1}(y)$. In [15, Example 4.3.1], the authors show that there does not exist a common quadratic function for both the open-loop system and the linear closed loop system (i.e. the system obtained by replacing $\tan ^{-1}(y)$ by $y$ in (18a)). However, using $V(x, \phi)$ as in (9) with $\operatorname{deg}\left(V_{0}(x, \phi), x\right)=4$ and the degrees of the other polynomials of Proposition 1 being two, we can prove the global stability of the non-linear closed-loop system. Suitable level sets of $V(x, \phi)$ depict the decrease of the function along the trajectories of the system in Figure 2.

\section{B. Numerical Example 2: Vehicle Stability}

The second example considers the application of the presented results to a physical system and involves the estimation of the maximal region of attraction of a vehicle model. Consider the following vehicle dynamics model from [16]

$$
\begin{aligned}
m \dot{v} & =-m u_{0} \omega+F_{f y}\left(y_{1}\right)+F_{r y}\left(y_{2}\right) \\
I \dot{\omega} & =-a F_{f y}\left(y_{1}\right)-b F_{r y}\left(y_{2}\right) \\
y_{1} & =\frac{v}{u_{0}}+\frac{a \omega}{u_{0}} \\
y_{2} & =\frac{v}{u_{0}}-\frac{b \omega}{u_{0}}
\end{aligned}
$$




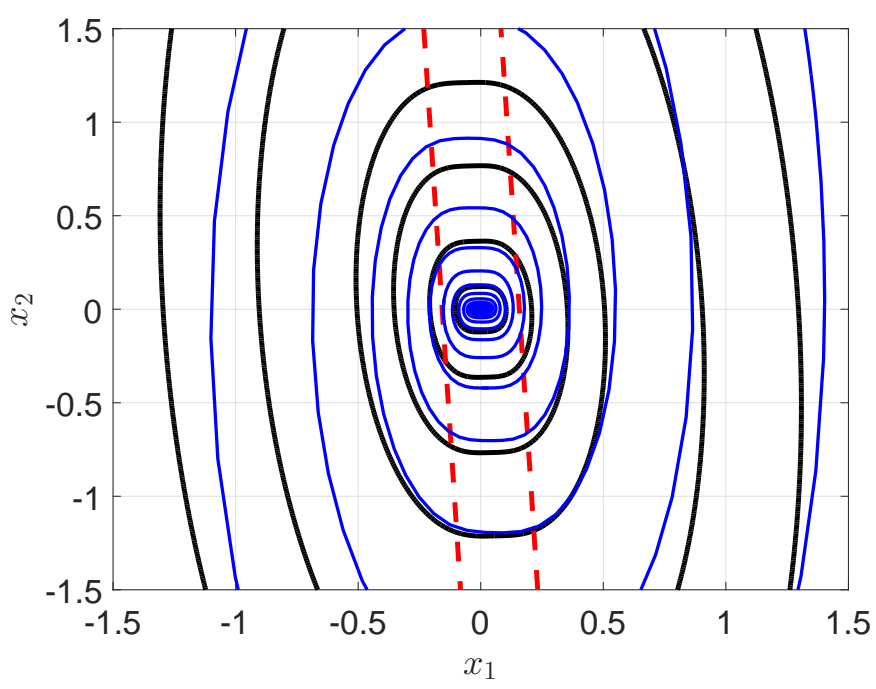

Figure 2. Convergence of the state trajectories of (18) (blue). Level sets of the computed function $V(x, \phi)$ (black) and lines corresponding to the set $\left\{x \in \mathbb{R}^{2}:\left|\tan ^{-1}(y(x))\right|=0.8\right\}$ (red) are also shown.

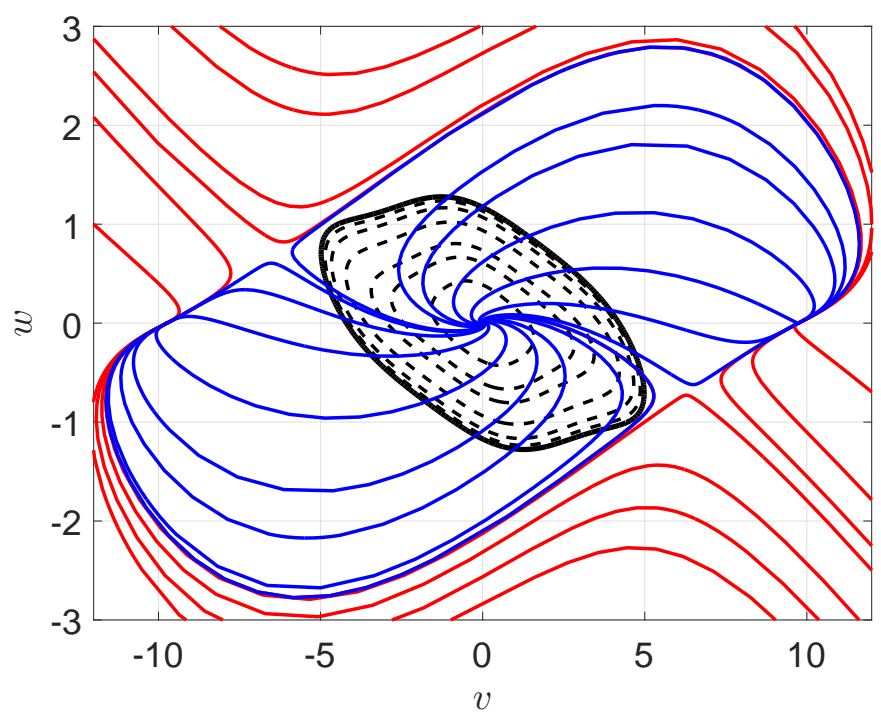

Figure 3. Estimate of the region of attraction $(\mathcal{E}(V, \rho))$ (black solid) of the vehicle model of numerical example 4 obtained using the polynomial Lyapunov function of Proposition 1. Also shown are sublevel sets of the Lyapunov function (black dashed), stable trajectories of the system (blue) and unstable trajectories (red).

with states lateral velocity $v$ and rotational velocity $\omega$. The model parameters are mass $m=2527 \mathrm{~kg}$, longitudinal speed $u_{0}=20 \mathrm{~m} \mathrm{~s}^{-1}$, moment of inertia $I=6550 \mathrm{~kg} \mathrm{~m}{ }^{2}$, mass centre from front axle $a=1.37 \mathrm{~m}$, mass centre from rear axle $b=1.86$.

The nonlinearity of the model enters via the tyre forces $F_{f y}\left(y_{1}\right)$ and $F_{r y}\left(y_{2}\right)$ given in [16] by

$$
\begin{gathered}
F_{f y}=-2 C_{f}\left(\phi_{1}\left(y_{1}\right)-K_{f} \phi_{1}\left(y_{1}\right)^{3}\right) \\
F_{r y}=-2 C_{r}\left(\phi_{2}\left(y_{2}\right)-K_{r} \phi_{2}\left(y_{2}\right)^{3}\right)
\end{gathered}
$$

where $C_{r}=C_{f}=57300 \mathrm{~N} \mathrm{rad}^{-1}$ are the cornering stiffnesses and $K_{f}=K_{r}=4.87$ are tyre force parameters. Compared to the "Magic Formula Tire Models" of [17,
Chapter 4], this definition of the tyre forces assumes that the tyre slips do not become large. Under the assumption of small steering angle deflection, the front and rear tyre lateral slip angles are

$$
\begin{aligned}
& \phi_{1}\left(y_{1}\right)=\tan ^{-1}\left(y_{1}\right), \\
& \phi_{2}\left(y_{2}\right)=\tan ^{-1}\left(y_{2}\right) .
\end{aligned}
$$

This vehicle model can be expressed as (1) with $D_{1}=1$, $B_{w}=0, C_{z}=0, \bar{D}_{2}=\left(1+y_{1}^{2}\right)\left(1+y_{2}^{2}\right)$,

$$
\begin{aligned}
C & =\left[\begin{array}{cc}
\frac{1}{u_{0}} & \frac{a}{u_{0}} \\
\frac{1}{u_{0}} & -\frac{b}{u_{0}}
\end{array}\right], \\
N_{1} & =\left[\begin{array}{c}
-u_{0} \omega \\
0
\end{array}\right] \\
& +\left[\begin{array}{llll}
-\frac{2 C_{f}}{m} & \frac{2 C_{f} K_{f}}{m} & -\frac{2 C_{r}}{m} & \frac{2 C_{r} K_{r}}{m} \\
\frac{2 a C_{f}}{I} & -\frac{2 a C_{f} K_{f}}{I} & -\frac{2 b C_{r}}{I} & \frac{2 b C_{r} K_{r}}{I}
\end{array}\right]\left[\begin{array}{c}
\phi_{1}\left(y_{1}\right) \\
\phi_{1}\left(y_{1}\right)^{3} \\
\phi_{2}\left(y_{2}\right) \\
\phi_{2}\left(y_{2}\right)^{3}
\end{array}\right], \\
\bar{N}_{2} & =\left[\begin{array}{ccc}
\left.1+y_{2}^{2}\right) & 0 \\
0 & \left(1+y_{1}^{2}\right)
\end{array}\right] .
\end{aligned}
$$

The region of attraction for this model obtained using Proposition 1 is shown in Figure 3. We have used $\operatorname{deg}\left(V_{0}(x, \phi), x\right)=8$ and with the other polynomial variables of Proposition 1 being of degree 2. Including the nonlinearities of the model directly within the stability analysis of the system in this manner will give a more representative picture of the stability profile of the real system compared to using a linear analysis, as is commonly used [17, Chapter 11].

\section{Conclusion}

This paper has considered the generalised stability problem with conditions being derived to verify stability and obtain minimal $\mathcal{L}_{2}$ gain bounds from disturbance to output. The class of nonlinear systems considered is, along with its Jacobian, a rational function of the states and sector bounded nonlinearities. The conditions were derived using a Lyapunov (storage) function that is polynomial with respect to the states and the nonlinearity and has a polynomially scaled integral term. The main result of the paper relaxes strict positivity of the terms of this Lyapunov function so as to reduce the conservatism of the conditions. As a consequence, the Lyapunov functions are not required to be sum-of-squares with respect to the nonlinearity and the scaling terms are also not required to be strictly positive. A convex formulation for the conditions was given such that they could be efficiently verified, as shown in two numerical examples.

A system of practical interest in the form (1) is the finite dimensional approximation of the electrochemical model of a supercapacitor [18]. In such a system, the (locally) sector bounded nonlinearity $\ln (1+a x)$ appears. We are currently extending the results of this paper to the problem of computing the $\mathcal{L}_{2}$ gain of the supercapacitor to estimate its resistance. Due to the relatively high dimension (20 
states) of the discretised supercapacitor model, a numerical solution of the optimization problem in Proposition 1 currently cannot be obtained.

\section{APPENDIX}

Definition 1: A multivariate polynomial $p(\theta)$ is said to be a sum-of-squares polynomial if there exists a finite set of polynomials $h_{i} \in \mathcal{R}[\theta]$ satisfying

$$
p(\theta)=\sum_{i=1}^{n} h_{i}^{2}(\theta) .
$$

The search for a sum-of-squares decomposition of a polynomial is affine in the coefficients of $h_{i}$ and as such can be expressed as a semi-definite programme (SDP) [13].

Proposition 2: A polynomial $p(\theta) \in \mathcal{R}[\theta]$ of degree $2 d$ is an sum-of-squares polynomial if and only if there exists $Q \in \mathbb{S}_{\geq 0}$ and a vector of monomials $Z(\theta) \in \mathcal{R}^{M}[\theta]$ such that

$$
p(\theta)=Z(\theta)^{T} Q Z(\theta)
$$

then $p(\theta)$ is sum-of-squares.

\section{REFERENCES}

[1] A. I. Lurie and V. N. Postnikov, "On the theory of stability of control system," Applied mathematics and mechanics, vol. 8(3), 1944.

[2] S. V. Gusev and A. Likhtarnikov, "Kalman-PopovYakubovich lemma and the S-procedure: a historical essay," Automation and Remote Control, vol. 67, no. 11, pp. 1768-1810, 2006.

[3] H. Khalil, Nonlinear Systems, 3rd ed. Prentice-Hall. Upper Saddle River, NJ, 2002.

[4] G. Valmorbida, R. Drummond, and S. R. Duncan, "Positivity conditions of Lyapunov functions for systems with slope restricted nonlinearities," in Proc. of the American Control Conference. Boston, MA, USA: IEEE, 2016, pp. 258-263.

[5] P. Park, "Stability criteria of sector- and sloperestricted Lur'e systems," IEEE Transactions on Automatic Control, vol. 47, no. 2, pp. 308-313, Feb 2002.

[6] M. C. Turner and M. Kerr, "Lyapunov functions and $\mathcal{L}_{2}$ gain bounds for systems with slope restricted nonlinearities," Systems $\&$ Control Letters, vol. 69, pp. $1-6,2014$.
[7] E. Hancock and A. Papachristodoulou, "Generalised absolute stability and sum of squares," Automatica, vol. 49, no. 4, pp. 960-967, 2013.

[8] G. Chesi, "Estimating the domain of attraction for non-polynomial systems via LMI optimizations," $A u$ tomatica, vol. 45, no. 6, pp. 1536-1541, 2009.

[9] S. Mastellone, P. Hokayem, C. Abdallah, and P. Dorato, "Nonlinear stability analysis for non-polynomial systems," in Proc. of the 22 $2^{\text {nd }}$ American Control Conference. Boston, MA: IEEE, 2004, pp. 17251730.

[10] A. Papachristodoulou and S. Prajna, "Analysis of non-polynomial systems using the sum of squares decomposition," in Positive Polynomials in Control. Springer, 2005, pp. 23-43.

[11] R. Drummond, G. Valmorbida, and S. R. Duncan, "Lyapunov analysis of nonlinear systems with rational vector field and Jacobian," in Proc. of the $11^{\text {th }}$ UK International Conference on Control. Belfast, UK: IEEE, 2016.

[12] J. Carrasco, M. Turner, and W. Heath, "ZamesFalb multipliers for absolute stability: From O'Shea's contribution to convex searches," European Journal of Control, vol. 28, pp. 1 - 19, 2016.

[13] G. Blekherman, P. A. Parrilo, and R. R. Thomas, Semidefinite optimization and convex algebraic geometry. SIAM. Philadelphia, PA., 2012.

[14] G. Valmorbida and J. Anderson, "Region of attraction estimation using invariant sets and rational Lyapunov functions," Automatica, vol. 75, pp. 37-45, 2017.

[15] L. Zaccarian and A. Teel, Modern anti-windup synthesis: control augmentation for actuator saturation. Princeton University Press. Princeton, NJ, 2011.

[16] M. Masouleh and D. Limebeer, "Non-linear vehicle domain of attraction analysis using sum-of-squares programming," in Proc. of the Conference on Control Applications. Buenos Aires, Argentina: IEEE, 2016, pp. 1209-1214.

[17] H. Pacejka, Tire and vehicle dynamics. $2^{\text {nd }}$ edition. Butterworth-Heinemann. Oxford, UK., 2005.

[18] R. Drummond, D. Howey, and S. Duncan, "Loworder mathematical modelling of electric double layer supercapacitors using spectral methods," Journal of Power Sources, vol. 277, pp. 317-328, 2015. 